\title{
Exploring the perceived barriers to following a Mediterranean style diet in women of childbearing age in the United Kingdom: a qualitative study
}

\author{
H. Kretowicz, V. Hundley and F. Tsofliou \\ Department of Human Sciences and Public Health, Faculty of Health and Social Sciences, Bournemouth University, \\ BH12 $5 B B$
}

Promotion of healthy dietary patterns similar to a Mediterranean (Med.) style diet in the preconception years has been suggested as a dietary strategy to prevent maternal obesity ${ }^{(1)}$. However, it is uncertain whether adoption is feasible in women of childbearing age in the United Kingdom (UK). The present qualitative study aimed to investigate the perceived barriers to following a Med. style diet in women of childbearing age in the UK.

Women were recruited from the South of England using social media, posters, email, and word of mouth (April - June 2017). Women were eligible if aged between 18 and 49 years, held UK residency, were nulliparous, did not study or have an occupation directly related to nutrition, and were shown to have low or medium adherence only to a Mediterranean style diet ${ }^{(2)}$ in eligibility screening. Data from semi-structured focus groups were used to generate deep insights; participants $(n=20$, mean age 26 years) took part in 6 focus groups. All interviews were digitally recorded, transcribed verbatim, and analysed using inductive thematic analysis ${ }^{(3)}$. Five main themes emerged:

i) Mediterranean diet features; overall, the varied content of a Med. style diet was considered appealing ("It's quite diverse...normally you're quite restricted but it seems like most food groups are involved in the diet").

ii) Perceived benefits; largely, participants were motivated to follow a healthy diet for disease prevention ("I want to prevent things before they're at risk, you think I don't want to have diabetes so I am going to change this...I am going to think about what I am eating") and/or aesthetic benefits ("it might even have to go down the route of 'you will have nice hair if you have this meal' or 'you will have good skin' and again focussing more on looks"). The perceived health benefits of a Med. style diet therefore could enable adoption, but the lack of highlighted aesthetic benefits pose as a potential barrier due to the importance placed on appearance in this group of women ("I probably should be but, I don't feel like things like heart disease and stroke... I don't think about that at the moment, I think more about fitness and what I am going to look like in a bikini"). Surprisingly, even though health benefits were enabling, promoting a Med. style diet to childbearing aged women for pregnancy and offspring benefits was perceived negatively ("You feel kind of like a baby making machine").

iii) Existing dietary behaviour and knowledge; these factors showed potential to be enabling or act as a barrier depending on individual circumstance ("I know in other circumstances where people are brought up where take-aways are a regular occurrence... to change from that to this sort of diet would be very difficult"). Partner or family diet was an important factor in the participants' ability to follow a Med. style diet, with some indicating that non-compliance from a partner would prevent personal adherence to the diet ("I would love to cook with lentils and pulses...but I would need to work on my other half, because he thinks a meal without meat is not a meal").

iv) Practical factors; these included cost, time, convenience, and meal preparation as possible barriers ("I know that if I was following a diet where every evening you had half an hour or an hour preparation...I would just come home and order pizza instead").

v) Information source; all participants expressed a preference for accessing information delivered via the internet, and possible online promotion of a Med. style diet was considered appealing ("It's so easy to find stuff online, from recipes to other ways to get more olive oil into your diet or whatever, there's never been a better time").

The present study highlighted that promotion of a Med. style diet in childbearing aged women in the UK is feasible, and the insights generated will be helpful in assisting the development of an intervention to promote Mediterranean style diet adoption.

1. Hanson M, Barker M, et al. (2017) Lancet Diabetes Endocrinol 5, 65-76.

2. Martínez-González MA, García-Arellano A, et al. (2012) PLoS One 8, e43134.

3. Braun V \& Clarke V (2006) Research in Psychology 3, 77-101. 